Pacific

Journal of

Mathematics

\title{
CLASSIFICATION OF MODULI SPACES \\ OF ARRANGEMENTS OF NINE PROJECTIVE LINES
}

FEI YE 


\title{
CLASSIFICATION OF MODULI SPACES OF ARRANGEMENTS OF NINE PROJECTIVE LINES
}

\author{
FEI YE
}

\begin{abstract}
In the study of line arrangements, searching for minimal examples of line arrangements whose fundamental groups are not combinatorially invariant is a very interesting and hard problem. It is known that such a minimal arrangement must have at least 9 lines. In this paper, we extend the number to 10 by a new method. We classify arrangements of 9 projective lines according to the irreducibility of their moduli spaces and show that fundamental groups of complements of arrangements of 9 projective lines are combinatorially invariant. The idea and results have been used to classify arrangements of 10 projective lines.
\end{abstract}

\section{Introduction}

A hyperplane arrangement $\mathscr{A}=\left\{L_{1}, L_{2}, \ldots, L_{n}\right\}$ in $\mathbb{C P}^{r}$ is a finite collection of hyperplanes. We call $M(\mathscr{A})=\mathbb{C P}^{r} \backslash\left(\bigcup_{L \in \mathscr{A}} L\right)$ the complement of $\mathscr{A}$. The set $L(\mathscr{A})=\left\{\bigcap_{i \in S} L_{i} \mid S \subseteq\{1,2, \ldots, n\}\right\}$ partially ordered by reverse inclusion is called the intersection lattice of $\mathscr{A}$. Let $\mathscr{A}_{1}$ and $\mathscr{A}_{2}$ be two arrangements of $n$ hyperplanes. We say that intersection lattices $L\left(\mathscr{A}_{1}\right)$ and $L\left(\mathscr{A}_{2}\right)$ are isomorphic, denoted by $L\left(\mathscr{A}_{1}\right) \sim L\left(\mathscr{A}_{2}\right)$, if there is a permutation $\phi$ of the set $\{1,2, \ldots, n\}$ such that

$$
\operatorname{dim} \bigcap_{\substack{i \in S \\ G_{i} \in \mathscr{A}_{1}}} G_{i}=\operatorname{dim} \bigcap_{\substack{j \in \phi(S) \\ H_{j} \in \mathscr{A}_{2}}} H_{j}
$$

for any nonempty subset $S \subseteq\{1,2, \ldots, n\}$. Two arrangements are lattice isomorphic if their lattices are isomorphic. In this paper, we only consider line arrangements in $\mathbb{C P} \mathbb{P}^{2}$.

An essential topic in hyperplane arrangements theory is to study the interaction between topology of complements and combinatorics of intersection lattices. Naturally enough, one may ask how close topology and combinatorics of a given arrangement

This work was partially supported by the Oswald Veblen Fund and by the Minerva Foundation of Germany.

MSC2010: 14N20, 32S22, 52C35.

Keywords: line arrangements, moduli spaces. 
are related. Two arrangements, $\mathscr{A}_{1}$ and $\mathscr{A}_{2}$, are homeomorphic equivalent if there is a homeomorphism between their complements. A more concrete question is: how close are lattice isomorphism and homeomorphic equivalence to being in one-to-one correspondence?

The deepest theorem in the theory of line arrangement in projective 2-dimensional space is that of Jiang and Yau [1998], which asserts that the intersection lattice of the line arrangement is a topological invariant. It is natural to ask to what extent the converse of the Jiang-Yau theorem is true. Jiang and Yau [1994], and subsequently Wang and Yau [2005], have shown that the converse statement is also true for a large class of line arrangements. Therefore, the Jiang-Yau theorem initiates a new research direction: Can one find a Zariski pair of line arrangements; that is, a pair of arrangements which are lattice isomorphic but not homeomorphic equivalent.

A pair of arrangements which are lattice isomorphisms but not homeomorphic equivalent is called a Zariski pair. Our definition is stronger than the definition introduced by Artal [1994], which we shall call weak Zariski pairs (see [Artal et al. 2008] for a survey on Zariski pairs). The first Zariski pair of arrangements was constructed by Rybnikov [2011]. Each arrangement in Rybnikov's example consists of 13 lines and 15 triple points. Artal et al. [2005] provided another (weak) Zariski pair of two arrangements $\mathscr{H}^{+}:=\mathscr{C}^{+} \cup\left\{N^{+}\right\}$and $\mathscr{H}^{-}:=\mathscr{C}^{-} \cup\left\{N^{-}\right\}$, where $\mathscr{C}^{+}, \mathscr{C}^{-}$ are arrangements (Figure 7) extending Falk-Sturmfels arrangements (Figure 2), and $N^{+}, N^{-}$are lines passing through a triple point and a double point of $\mathscr{C}^{ \pm}$. The proof is based on the observation that there is no order-preserving homeomorphism between $\left(\mathbb{P}^{2}, \mathscr{C}^{+}\right)$and $\left(\mathbb{P}^{2}, \mathscr{C}^{-}\right)$. In the contrary direction, Garber, Teicher, and Vishne [Garber et al. 2003] proved that there is no Zariski pair of arrangements of up to 8 real lines which covered the result of Fan [1997] on arrangements of 6 lines. This result was recently generalized to arrangements of 8 complex lines by Nazir and Yoshinaga [2012].

A natural question is: what is the minimal number of lines of a Zariski pair of line arrangements?

On the other hand, it was Jiang and Yau [1994] who first observed that the statement "two lattice isotopy line arrangements (that is, they are connected by a one-parameter family with constant intersection lattice) have diffeomorphic complements" follows trivially from Teissier's numerical characterization of the Whitney condition. In [Jiang and Yau 1994] and [Wang and Yau 2005], the authors found large classes of line arrangements, called nice arrangements and simple arrangements, whose intersection lattices determine the topology of the complements. Nazir and Yoshinaga [2012] found new classes of line arrangements whose intersection lattices determine the topology of the complements. Unlike nice and simple arrangements whose intersection lattices have special properties, Nazir and Yoshinaga's new classes require that all intersection points with multiplicity at 
least 3 be in special positions. This makes their results more useful for studying arrangements of a few lines. Indeed, in their paper they classify arrangements of 8 lines and give a list of classes of arrangements of 9 lines.

In this paper, we introduce new ideas to classify arrangements of lines. We prove that Nazir and Yoshinaga's list on the classification of arrangements of 9 lines is complete. As a corollary, we conclude that there is no Zariski pair of arrangements of 9 lines. The idea and results of this paper have been used to classify moduli spaces of arrangements of 10 projective lines (see [Amram et al. 2012]).

The paper is organized as follows: In Section 2, we recall results in Nazir and Yoshinaga. In Section 3, we prove that their list of classes of arrangements of 9 lines is complete. In Section 4, we consider the example of arrangements of 10 lines $\mathscr{C}^{ \pm}$and give an explicit diffeomorphism between the complements $M\left(\mathfrak{C}^{ \pm}\right)$.

\section{Simple $C_{\leq 3}$ line arrangements}

Consider the dual space $\left.(\mathbb{C P})^{2}\right)^{*}$ of the projective space $\mathbb{C P} \mathbb{P}^{2}$. A line arrangement $\mathscr{A}=\left\{L_{1}, L_{2}, \ldots, L_{n}\right\}$ can be viewed as an $n$-tuple of points $\left(L_{1}^{*}, L_{2}^{*}, \ldots, L_{n}^{*}\right)$ in the product of the dual spaces $\left(\left(\mathbb{C P}^{2}\right)^{*}\right)^{n}$. We define the moduli space of arrangements with the fixed lattice $L(\mathscr{A})$ as

$$
\mu_{\mathscr{A}}=\frac{\left\{\mathscr{B} \in\left(\left(\mathbb{C P}^{2}\right)^{*}\right)^{n} \mid L(\mathscr{B}) \sim L(\mathscr{A})\right\}}{\mathrm{PGL}_{3}(\mathbb{C})} \subseteq \frac{\left(\left(\mathbb{C P}^{2}\right)^{*}\right)^{n}}{\mathrm{PGL}_{3}(\mathbb{C})}
$$

We say that a singular point $P$ of $L_{1} \cup L_{2} \cup \cdots \cup L_{n}$ is a multiple point of $\mathscr{A}$ if the multiplicity of $P$ is at least 3 .

The following definition is a combination of Nazir and Yoshinaga's original definitions of $C_{1}, C_{2}$, and simple $C_{3}$ arrangements.

Definition 2.1. A line arrangement is called $C_{\leq 3}$ if all the multiple points are on at most three lines; say, $L_{1}, L_{2}$, and $L_{3}$. A line arrangement is called simple $C_{\leq 3}$ if it is $C_{\leq 3}$, and one of the following condition holds:

(i) $L_{1} \cap L_{2} \cap L_{3} \neq \varnothing$, or

(ii) one of $L_{1}, L_{2}$ and $L_{3}$ contains at most one more multiple point apart from the possible multiple points $L_{1} \cap L_{2}, L_{2} \cap L_{3}$, and $L_{1} \cap L_{3}$.

Here are some examples of arrangements which are not simple $C_{\leq 3}$ :

Example 2.2. A Mac Lane arrangement (see Figure 1) consists of eight lines and eight triple points such that each line passes through exactly three triple points. It is not hard to check that the moduli space of Mac Lane arrangements consists of two points. Representatives of the two points can be defined by the equation

$$
x y(x-z)(y-z)(x-y)\left(x-\varepsilon^{ \pm} z\right)\left(y-\varepsilon^{ \pm} z\right)\left(-\varepsilon^{\mp} x-y+z\right)=0,
$$




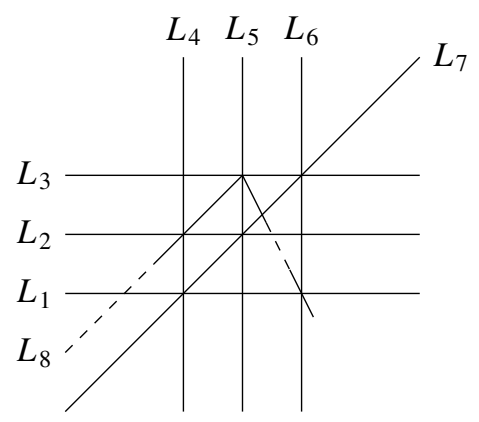

Figure 1. A Mac Lane arrangement.

where $\varepsilon^{ \pm}=\frac{1}{2}(1 \pm \sqrt{-3})$ are the roots of $x^{2}-x+1=0$.

Since each line passes through three triple points, there are at most seven triple points on three lines. Thus, Mac Lane arrangements cannot be simple $C_{\leq 3}$.

Example 2.3. Falk-Sturmfels arrangements are the arrangements of nine lines with one quadruple point, eight triple points, and one line passing through four triple points (Figure 2). We denote them by

$$
F S^{ \pm}=\left\{L_{i}^{ \pm}, K_{i}^{ \pm}, H_{9}^{ \pm}, i=1,2,3,4\right\},
$$

where the lines are defined by

$$
\begin{aligned}
& L_{1}^{ \pm}: x=0, \quad L_{2}^{ \pm}: x=\gamma_{ \pm}(y-z), \quad L_{3}^{ \pm}: y=z, \quad L_{4}^{ \pm}: x+y=z, \\
& K_{1}^{ \pm}: x=z, \quad K_{2}^{ \pm}: x=\gamma_{ \pm} y, \quad K_{3}^{ \pm}: y=0, \quad K_{4}^{ \pm}: x+y=\left(\gamma_{ \pm}+1\right) z, \\
& H_{9}^{ \pm}: z=0 \text {, }
\end{aligned}
$$

with $\gamma_{ \pm}=\frac{1}{2}(1 \pm \sqrt{5})$ the roots of $x^{2}-x-1=0$. It is known (see [Nazir and

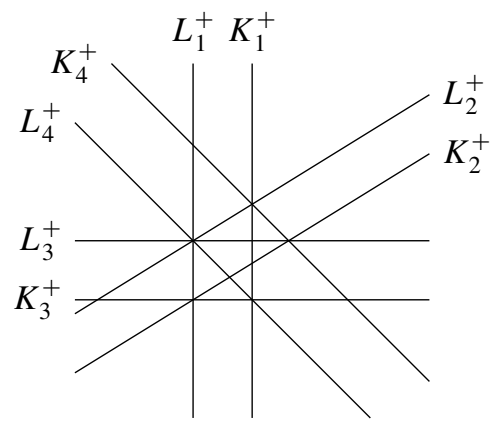

$F S^{+}$

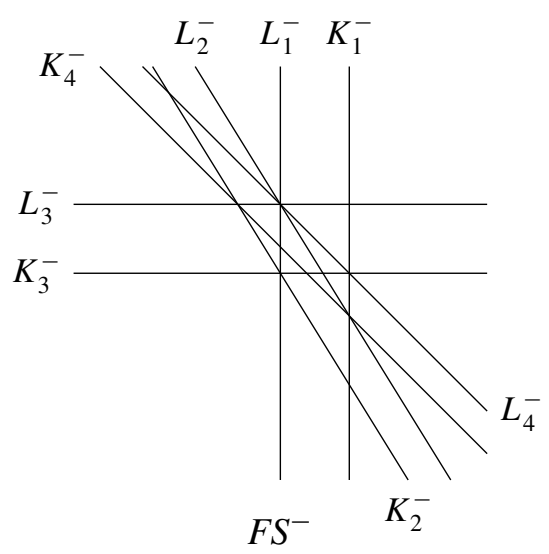

Figure 2. Falk-Sturmfels arrangements. 


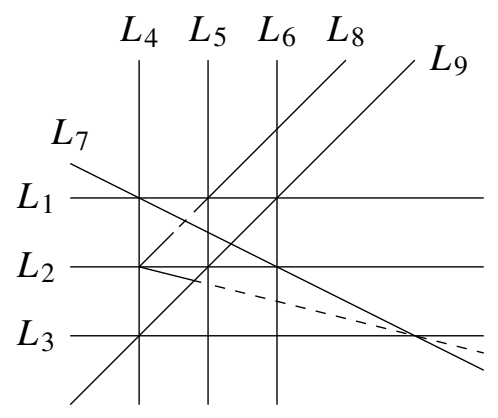

Figure 3. The arrangements $\mathscr{A}^{ \pm \sqrt{-1}}$.

Yoshinaga 2012, Example 5.2], for instance) that the moduli space $\mu_{L\left(F S^{ \pm}\right)}$consists of 2 points, $\left\{F S^{+}, F S^{-}\right\}$.

Example 2.4 [Nazir and Yoshinaga 2012, Example 5.3]. The arrangements $\mathscr{A}^{ \pm \sqrt{-1}}$ consist of nine lines and ten triple points such that there are three lines which do not intersect at a point and have four triple points on each. Moreover, each of the other six lines contains exactly three triple points. Those arrangements (see Figure 3 ) can be defined by the equation

$x y(x-z)(y-z)(x \mp \mathrm{i} z)(y \mp \mathrm{i} z)(x-y)(( \pm \mathrm{i}-1) x \pm \mathrm{i} y+z)((1 \mp \mathrm{i}) x+y-z)=0$.

Lemma 2.5 [Nazir and Yoshinaga 2012, Lemma 4.4]. If a line arrangement is not simple $C_{\leq 3}$, then it has 6 lines $L_{1}, L_{2}, \ldots, L_{6}$ such that $L_{1} \cap L_{2} \cap L_{3} \neq \varnothing$, $L_{4} \cap L_{5} \cap L_{6} \neq \varnothing$, and $\left(L_{1} \cup L_{2} \cup L_{3}\right) \cap\left(L_{4} \cup L_{5} \cup L_{6}\right)$ consists of 9 distinct double points.

Let $\mathscr{A}_{s}=\left\{L_{1}, L_{2}, \ldots, L_{6}\right\}$ be the arrangement which has two triple points $L_{1} \cap L_{2} \cap L_{3}$ and $L_{4} \cap L_{5} \cap L_{6}$, and nine double points $Q_{i j}=L_{i} \cap L_{j+3}$, where $i, j \in\{1,2,3\}$.

Using Lemma 2.5, one can easily prove that an arrangement of 7 lines is simple $C_{\leq 3}$. It is also not hard to prove the following result:

Proposition 2.6 [Nazir and Yoshinaga 2012, Proposition 4.6]. An arrangement of eight lines is either a simple $C_{\leq 3}$ line arrangement or a Mac Lane arrangement.

More generally:

Theorem 2.7 [Nazir and Yoshinaga 2012, Theorem 3.5]. The moduli space $M_{\mathscr{A}}$ of simple $C_{\leq 3}$ line arrangements with the fixed intersection lattice $L(\mathscr{A})$ is irreducible.

Let $\mathscr{A}=\left\{L_{1}, L_{2}, \ldots, L_{n}\right\}$ be a line arrangement, and $\mathscr{A}^{\prime}=\left\{L_{1}, L_{2}, \ldots, L_{n-1}\right\}$ be a subarrangement. The following lemma shows when the irreducibility of the moduli space $M_{\mathscr{A}^{\prime}}$ will be inherited: 
Lemma 2.8 [Nazir and Yoshinaga 2012, Lemma 2.4]. Assume that the line $L_{n}$ passes through at most two multiple points of the arrangement $\mathscr{A}$. Then the moduli space $\mathcal{M}_{\mathscr{A}}$ is a fiber bundle over the moduli space of $\mathcal{M}_{\mathscr{A}}$. In particular, the moduli space $\mathcal{M}_{\mathscr{A}}$ is irreducible if $\mathcal{M}_{\mathscr{A}^{\prime}}$ is irreducible.

Applying this lemma to arrangements of 9 lines, we have the following corollary:

Corollary 2.9. Let $\mathscr{A}$ be an arrangement of 9 lines. If there is a line in $A$ which passes through at most two multiple points of $\mathscr{A}$, then either $\mathscr{A}$ contains a Mac Lane arrangement as a subarrangement, or the moduli space $M_{\triangle}$ is irreducible.

Proof. The conclusion follows directly from Proposition 2.6 and Lemma 2.8.

\section{Classification of arrangements of 9 lines}

For a line arrangement $\mathscr{A}$, we denote by $m_{\mathscr{A}}$ the highest multiplicity of a multiple point of $\mathscr{A}$. We will divide the classification of arrangements of 9 lines into three cases according to the value of $m_{\mathscr{A}}$.

Let $n_{r}$ be the number of multiple points of multiplicity $r$. We first recall two well-known results on the number of multiple points.

Theorem 3.1 [Hirzebruch 1986]. Let $\mathscr{A}$ be an arrangement of $t$ lines in $\mathbb{C P}^{2}$. Assume that $n_{t}=n_{t-1}=n_{t-2}=0$. Then,

$$
n_{2}+\frac{3}{4} n_{3} \geq t+\sum_{r \geq 5}(2 r-9) n_{r} .
$$

Lemma 3.2 (see, for instance, [Hirzebruch 1986]). Let $A$ be a line arrangement of $n$ lines in $\mathbb{C P}^{2}$. We have the intersection formula

$$
\frac{n(n-1)}{2}=\sum_{r \geq 2}\left(n_{r} \cdot \frac{r(r-1)}{2}\right) .
$$

\section{A. The case $m_{\mathscr{A}} \geq 5$.}

Proposition 3.3. Let $\mathscr{A}$ be an arrangement of 9 lines. If $\mathscr{A}$ has multiple points of multiplicity (at least 5), then the moduli space $\mathcal{M}_{\unlhd}$ is irreducible.

Proof. Assume that $L_{1} \cap L_{2} \cap \cdots \cap L_{5} \neq \varnothing$. There are at most 6 double points in $L_{6} \cup L_{7} \cup L_{8} \cup L_{9}$. Then, there are at most 7 multiple points in $L_{1} \cup L_{2} \cup \cdots \cup L_{5}$. So, at least one of the five lines $L_{1}, L_{2}, \ldots, L_{5}$ contains only two multiple points. By Corollary 2.9, the moduli space $M_{\mathscr{A}}$ is irreducible.

3B. The case $\boldsymbol{m}_{\mathscr{A}}=4$. Let $\mathscr{A}$ be an arrangement of 9 lines. In this subsection, we assume that multiple points of $\mathscr{A}$ are at most quadruple points. 
Proposition 3.4. Assume that each line of $A$ passes through at least three multiple points, and $n_{4} \geq 1$. Then either $\mathcal{M}_{\mathscr{A}}$ is irreducible, or $\mathscr{A}$ is lattice isomorphic to a Falk-Sturmfels arrangement.

Proof. We will first show that $n_{4}=1$.

Let $L_{1} \cap L_{2} \cap L_{3} \cap L_{4}$ be a quadruple point of $\mathscr{A}$. Since each line passes through at least three multiple points, $L_{1}, L_{2}, L_{3}$ and $L_{4}$ should pass through two more multiple points besides the quadruple point $L_{1} \cap L_{2} \cap L_{3} \cap L_{4}$. Then, there will be at least 9 multiple points on those four lines. Since multiple points of $\mathscr{A}$ are at most quadruple points, there are $n_{4}$ quadruple points. Therefore, there should be at least $9-n_{4}$ triple points on those four lines such that each line passes through at least 3 multiple points. By Theorem 3.1 and Lemma 3.2, we have

$$
36=6 n_{4}+3 n_{3}+n_{2} \geq 6 n_{4}+\frac{9}{4} n_{3}+9 \geq 6 n_{4}+\frac{9}{4}\left(9-n_{4}\right)+9 .
$$

Solving the inequality, we obtain that $n_{4} \leq \frac{9}{5}<2$. Therefore, by the assumption, we have $n_{4}=1$.

Now we claim that all triple points should be on the lines passing through the quadruple point.

Let $L_{1} \cap L_{2} \cap L_{3} \cap L_{4}$ be the quadruple. Suppose, contrary to our claim, that $L_{5} \cap L_{6} \cap L_{7}$ is a triple point which is not on $L_{1} \cup L_{2} \cup L_{3} \cup L_{4}$. Note that there are at most 7 double points on $L_{5} \cup L_{6} \cup L_{7} \cup L_{8} \cup L_{9}$. Then the intersection set $\left(L_{1} \cup L_{2} \cup L_{3} \cup L_{4}\right) \cap\left(L_{5} \cup L_{6} \cup L_{7} \cup L_{8} \cup L_{9}\right)$ will contain at most 7 triple points which are on $L_{1} \cup L_{2} \cup L_{3} \cup L_{4}$. However, there should be at least 8 triple points so that each of the four lines $L_{1}, L_{2}, L_{3}$ and $L_{4}$ passes through at least three multiple points. Therefore, by the assumption, all triple points must be on the lines passing through the quadruple point.

If $\mathscr{A}$ is simple $C_{\leq 3}$, then the moduli space $M_{\mathscr{A}}$ is irreducible. We only need to consider the case that $\mathscr{A}$ is not simple $C_{\leq 3}$. By Lemma 2.5, we know that the arrangement $\mathscr{A}$ has a subarrangement $\mathscr{A}_{s}$. It is not hard to see that the quadruple point should be $Q_{i j}$, where $i, j \in\{1,2,3\}$.

Up to a lattice isomorphism, we may assume that the only quadruple point is $L_{1} \cap L_{4} \cap L_{7} \cap L_{8}=Q_{11}$.

Since all triple points should be on $L_{1} \cup L_{4} \cup L_{7} \cup L_{8}$, then all possible triple points on $L_{7}$ and $L_{8}$ should be in the following set of points:

$$
\left\{Q_{22}, Q_{23}, Q_{32}, Q_{33}, L_{7} \cap L_{9}, L_{8} \cap L_{9}\right\} \text {. }
$$

The following figure is an example, but an excluding one, for $L_{6}$ passes through only one triple point.

Hence, each of the lines $L_{7}$ and $L_{8}$ will have at least one $Q_{i j}$, where $i, j \in\{2,3\}$. 


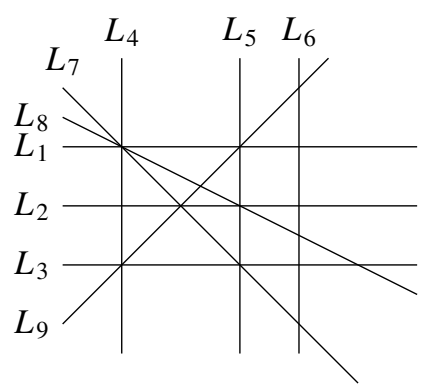

Figure 4. An excluding arrangement.

(1) Assume that each of the lines $L_{7}$ and $L_{8}$ passes through exactly one of the points $\left\{Q_{22}, Q_{23}, Q_{32}, Q_{33}\right\}$.

If those two $Q_{i j}$ are on the same line, then one of the four lines $L_{2}, L_{3}, L_{5}$ and $L_{6}$ will have at most two multiple points. For example, in Figure 4, the line $L_{6}$ passes through only one multiple point, $L_{4} \cap L_{5} \cap L_{6}$.

Assume that they are not on the same line. Up to switching labels between $L_{2}$ and $L_{3}$, correspondingly $L_{5}$ and $L_{6}$, we may assume that $Q_{32} \in L_{7}$ and $Q_{23} \in L_{8}$. Then, either $\left\{Q_{31}, Q_{13}\right\} \subset L_{9}$ or $\left\{Q_{21}, Q_{12}\right\} \subset L_{9}$. Correspondingly, $\left\{L_{2} \cap L_{7}, L_{5} \cap L_{8}\right\} \subset$ $L_{9}$ or $\left\{L_{3} \cap L_{7}, L_{6} \cap L_{8}\right\} \subset L_{9}$. By switching the labels between $L_{2}$ and $L_{3}, L_{5}$ and $L_{6}$, and $L_{7}$ and $L_{8}$, we see that those two arrangements are lattice isomorphic. Moreover, one can check that both arrangements (see Figure 5, left) are lattice isomorphic to Falk-Sturmfels arrangements.

(2) Assume that either the line $L_{7}$ or $L_{8}$ passes through two points out of the four points $Q_{22}, Q_{23}, Q_{32}$ and $Q_{33}$, but the other one passes through only one point out of the four points $Q_{22}, Q_{23}, Q_{32}$ and $Q_{33}$.

Up to a lattice isomorphism, we may assume that $\left\{Q_{11}, Q_{22}, Q_{33}\right\} \subset L_{7}$, and $\left\{Q_{11}, Q_{32}\right\} \subset L_{8}$. Then either $L_{2} \cap L_{8} \in L_{9}$, or $L_{6} \cap L_{8} \in L_{9}$. Otherwise, $L_{8}$ will have only two multiple points. Correspondingly, $\left\{Q_{31}, Q_{13}\right\} \subset L_{9}$, or $\left\{Q_{21}, Q_{12}\right\} \subset L_{9}$.
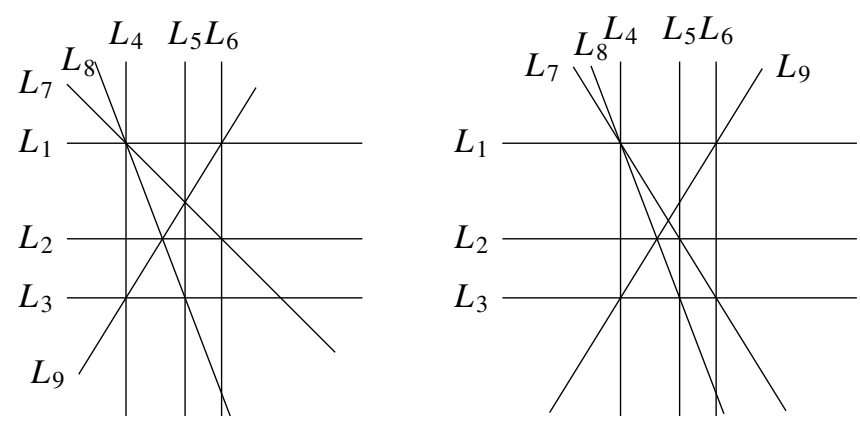

Figure 5. Falk-Sturmfels arrangements 1 and 2. 
By first switching the labels between $L_{1}$ and $L_{4}, L_{2}$ and $L_{5}$, and $L_{3}$ and $L_{6}$, then switching the labels between $L_{2}$ and $L_{3}$, and $L_{5}$ and $L_{6}$, we see that those two arrangements are lattice isomorphic. Moreover, we check that $\mathscr{A}$ (see Figure 5, right) is also lattice isomorphic to Falk-Sturmfels arrangements.

(3) Assume that $L_{7}$ and $L_{8}$ each contain two of $\left\{Q_{22}, Q_{23}, Q_{32}, Q_{33}\right\}$, then $L_{9}$ will contain at most two multiple points.

Therefore, we conclude that either $M_{\mathscr{A}}$ is irreducible or $\mathscr{A}$ is lattice isomorphic to a Falk-Sturmfels arrangement.

3C. The case $\boldsymbol{m}_{\mathscr{A}}=3$. Now we consider the last case in which all multiple points are triple points. We will first investigate possible values of $n_{3}$ such that each line has at least three triple points. Notice that $n_{3}$ should be no less than 9 . On the other hand, we observe the following result:

Lemma 3.5. Let $A$ be an arrangement of 9 lines, all of whose multiple points are triple points. Assume that $\mathcal{A}$ does not contain a Mac Lane arrangement as a subarrangement and is not simple $C_{\leq 3}$. Then $A$ has at most 10 triple points.

Proof. By Lemma 3.2, to show that $n_{3} \leq 10$, it is enough to show that $n_{2} \geq 4$.

Since $\mathscr{A}$ does not contain a Mac Lane arrangement, at most one of the lines $L_{7}$, $L_{8}$, and $L_{9}$ passes through three $Q_{i j}$, where $i, j \in\{1,2,3\}$ (defined as above). We may assume that each of the lines $L_{7}$ and $L_{8}$ passes through at most two $Q_{i j}$. By our assumption and Lemma 2.5 , the arrangement $\mathscr{A}$ has a subarrangement $\mathscr{A}_{s}$.

Let $x$ be the number of $Q_{i j}$ which are not in $L_{7} \cup L_{8} \cup L_{9}$. It is clear that $x \geq 2$. Let $y$ and $z$ be the number of double points of $\mathscr{A}$ which are in $L_{7} \cap\left(L_{1} \cup L_{2} \cup \cdots \cup L_{6}\right)$ and $L_{8} \cap\left(L_{1} \cup L_{2} \cup \cdots \cup L_{6}\right)$ respectively. If $y+z \geq 2$, then we have $n_{2} \geq x+(y+z) \geq 4$.

Assume that $y+z \leq 1$. Then each of the lines $L_{7}$ and $L_{8}$ should pass through exactly two $Q_{i j}$. Moreover, $L_{7} \cap L_{8}$ must be a triple point in $L_{1} \cup L_{2} \cup \cdots \cup L_{6}$. We see now the subarrangement $\mathscr{A}^{\prime}=\left\{L_{1}, L_{2}, \ldots, L_{8}\right\}$ has 7 double points. Without a loss of generality, we assume that $L_{7} \cap L_{8}$ is on $L_{2}$. It is not hard to see that the 7 double points of $\mathscr{A}^{\prime}$ are all on $L_{4} \cup L_{5} \cup L_{6}$. The line $L_{9}$ can only pass through at most three double points of $\mathscr{A}^{\prime}$. Therefore, the arrangement $\mathscr{A}$ still has at least 4 double points.

Remark 3.6. By Theorem 2.15 in [Csima and Sawyer 1993], if our arrangements are real arrangements, that is, if the coefficients of the defining equations of the lines are real numbers, then there are at least $\frac{60}{13}>4$ double points. Hence, there should be at most 10 triple points. However, there seems to be no similar result for complex line arrangements.

Proposition 3.7. Let $\mathscr{A}$ be an arrangement of 9 lines with 9 triple points. Assume that all multiple points of $\mathscr{A}$ are triple points, and each line passes through exactly three triple points. Then, the moduli space $M_{\mathscr{A}}$ is irreducible. 


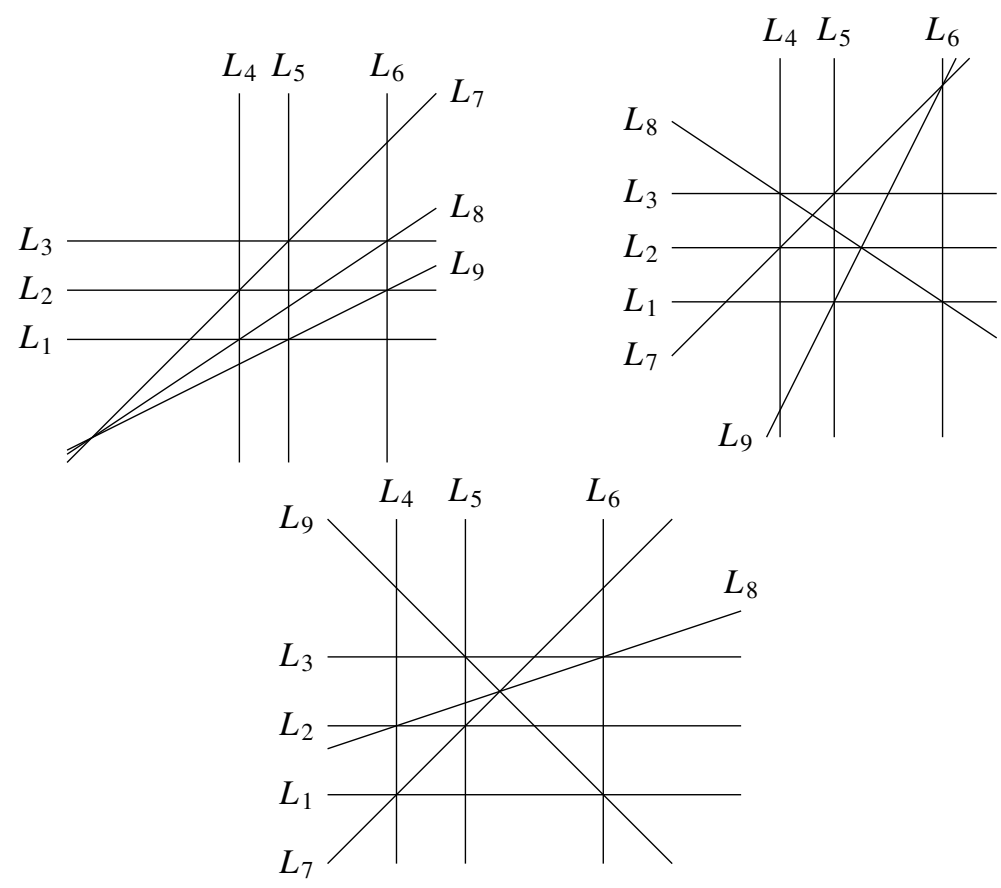

Figure 6. $9_{3}$ arrangements.

Proof. By Theorem 2.2.1 in [Grünbaum 2009] that $\mathscr{A}$ is lattice isomorphic to one of the three arrangements appearing in Figure 6.

One can check that the moduli space $M_{\mathscr{A}}$ is irreducible in each case.

Proposition 3.8. Let $\mathscr{A}$ be an arrangement of 9 lines with 10 triple points. Assume that all multiple points of $\mathscr{A}$ are triple points and each line passes through at least three triple points. If $\mathscr{A}$ is not simple $C_{\leq 3}$, then it is isomorphic to $\mathscr{A}^{ \pm \sqrt{-1}}$ (Figure 3).

Proof. Let $a$ be the number of lines that pass through 4 triple points and $b$ the number of lines that pass through 3 triple points. Then $a+b=9$ and $4 a+3 b=30$. We have $a=3$ and $b=6$.

If the three lines with 4 triple points on each of them intersect at a triple point, then all 10 triples should be on them. Consequently, the arrangement is simple $C_{\leq 3}$.

Assume that $L_{1}, L_{2}$ and $L_{4}$ are the three lines with 4 triple points on each of them and $L_{1} \cap L_{2} \cap L_{4}=\varnothing$. Then, at least two of $\left\{L_{1} \cap L_{2}, L_{1} \cap L_{4}, L_{2} \cap L_{4}\right\}$ are triple points. Otherwise there should be at least 11 triple points so that $L_{1}$, $L_{2}$, and $L_{4}$ will have 4 triple points. So, we may assume that $L_{1} \cap L_{2} \cap L_{3}$ and $L_{1} \cap L_{4} \cap L_{7}$ are triple points. Let $L_{4} \cap L_{5} \cap L_{6}$ be a triple point which is not on $L_{1} \cup L_{2} \cup L_{3}$. Then, $L_{7}$ must pass through $L_{2} \cap L_{5}$ or $L_{2} \cap L_{6}$. Otherwise, $L_{2}$ will have at most 3 triples. By switching labels of $L_{5}$ and $L_{6}$, we may assume that 
$L_{2} \cap L_{6} \cap L_{7} \neq \varnothing$. Then the two points $Q_{21}$ and $Q_{22}$ must be on $L_{8} \cup L_{9}$ so that $L_{2}$ will pass through 4 triple points. We may assume that $Q_{21} \in L_{8}$ and $Q_{22} \in L_{9}$. Since the line $L_{4}$ also passes through 4 triple points, then $Q_{31}$ should be on $L_{9}$. Similarly, since the line $L_{1}$ passes through 4 triple points, then $Q_{13}$ should be on $L_{9}$ and $Q_{12}$ should be on $L_{8}$. Now we have 9 triple points. The last triple point must be $L_{3} \cap L_{7} \cap L_{8}$ so that $L_{7}$ will pass through three triple points. The arrangements with such intersection lattices are just $\AA^{ \pm \sqrt{-1}}$ (see Figure 3).

3D. Classification and applications. We summarize Section 3 so far as follows: Theorem 3.9. Any arrangement of nine lines in $\mathbb{C P}^{2}$ belongs to one of the following classes:

(i) arrangements whose moduli spaces are irreducible;

(ii) arrangements containing Mac Lane arrangements (Example 2.2);

(iii) Falk-Sturmfels arrangements (Example 2.3);

(iv) $\mathscr{A}^{ \pm \sqrt{-1}}$ arrangements (Example 2.4).

Proof. The classification simply follows from Corollary 2.9 and Propositions 3.3, 3.4, 3.7, and 3.8.

As an application, we obtain the following result which generalizes a result of Theorem 8.3 in [Garber et al. 2003].

Theorem 3.10. The fundamental group of the complement of an arrangement of 9 lines is determined by the intersection lattice.

Proof. If the moduli space is irreducible, then the fundamental group is determined by the lattice according to the lattice-isotopy theorem.

It follows from Example 5.2 in [Nazir and Yoshinaga 2012] (see also Section 7.5 in [Cohen and Suciu 1997]) that the fundamental groups $\pi_{1}\left(M\left(F S^{+}\right)\right)$and $\pi_{1}\left(M\left(F S^{-}\right)\right)$are isomorphic. Let $\mathscr{A}_{1}$ and $\mathscr{A}_{2}$ be two arrangements containing Mac Lane arrangements. Then, either they are in the same connected component of the moduli spaces, or $\mathscr{A}_{1}$ and the conjugate of $\mathscr{A}_{2}$ are in the same connected component. By Theorem 3.9 in [Cohen and Suciu 1997], the fundamental groups of $\mathscr{A}_{1}$ and $\mathscr{A}_{2}$ are isomorphisms. According to the same theorem, the fundamental groups of $\mathscr{A}^{+\sqrt{-1}}$ and $\mathscr{A}^{-\sqrt{-1}}$ are isomorphic too.

\section{Arrangements of 10 lines: an example}

We have seen that there is no Zariski pair of arrangements of 9 lines, but we do not know if there is a Zariski pair of arrangements of 10 lines. To get a Zariski pair, a naive idea is to add lines to those arrangements whose moduli spaces are disconnected. In general, it is very hard to determine if the resulting pair of arrangements is a Zariski pair. The following example is a trial: 


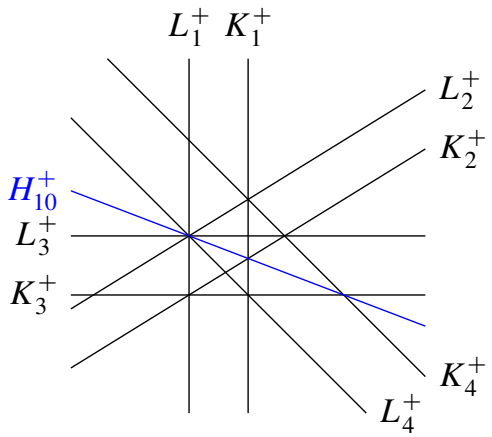

$\widetilde{F S}^{+}$

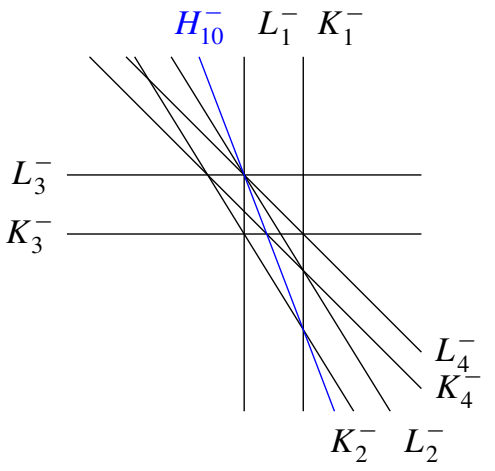

$\widetilde{F S}^{-}$

Figure 7. Extended Falk-Sturmfels arrangement.

Example 4.1. Starting from the Falk-Sturmfels arrangements (see Example 2.3), we will construct new arrangements of 10 lines such that the moduli space is disconnected.

We define two line arrangements of 10 lines, called extended Falk-Sturmfels arrangements (see Figure 7):

$$
\widetilde{F S}^{ \pm}=\left\{L_{i}^{ \pm}, K_{i}^{ \pm}, H_{9}^{ \pm}, H_{10}^{ \pm}, i=1,2,3,4\right\}
$$

by adding lines:

$$
H_{10}^{ \pm}: y=\left(\frac{1}{\gamma_{ \pm}}-1\right) x+z
$$

to $F S^{ \pm}$respectively.

Notice that $\widetilde{F S}^{ \pm}$are both fiber-type line arrangements according to Theorem 3.12 in [Jiang et al. 2001] .

It is not hard to see that $M_{\widetilde{F S}^{ \pm}} \cong M_{F S^{ \pm}}$. In fact, the line $H_{10}^{+}$(respectively, $H_{10}^{-}$) is always passing through three points of $L\left(F S^{ \pm}\right): L_{1}^{+} \cap L_{2}^{+}, K_{1}^{+} \cap K_{2}^{+}$and $K_{3}^{+} \cap K_{4}^{+}$ (respectively, $K_{2}^{-} \cap K_{4}^{-}, K_{3}^{-} \cap K_{4}^{-}$and $K_{1}^{-} \cap K_{2}^{-}$).

This pair of arrangements has been studied by Artal, Carmona, Cogolludo, and Marco. They show (Theorem 4.19 in [Artal et al. 2005]) that there is no orderpreserving homeomorphism between the pairs $\left(\mathbb{P}^{2}, \widetilde{F S}^{+}\right)$and $\left(\mathbb{P}^{2}, \widetilde{F S}^{-}\right)$. Here, we present an explicit diffeomorphism between the complements $M\left(\widetilde{F S}{ }^{+}\right)$and $M\left(\widetilde{F S}^{-}\right)$. In fact, by Example 5.2 in [Nazir and Yoshinaga 2012], we know that there is an automorphism $A \in \operatorname{PGL}\left(\mathbb{C}^{3}\right)$ of $\mathbb{C P}^{2}$,

$$
A:=\left(\begin{array}{rrr}
-\gamma_{-} & -1 & 0 \\
-\gamma_{-} & 0 & 0 \\
\gamma_{-} & 1 & 1
\end{array}\right),
$$


acting from the right (via matrix multiplication) on points $[x, y, z]$ in the projective space $\mathbb{P}^{2}$, which sends

$$
\begin{array}{rlll}
L_{1}^{+} \mapsto L_{3}^{-}, & L_{2}^{+} \mapsto L_{4}^{-}, & L_{3}^{+} \mapsto L_{2}^{-}, & L_{4}^{+} \mapsto L_{1}^{-}, \\
K_{1}^{+} \mapsto K_{3}^{-}, & K_{2}^{+} \mapsto K_{4}^{-}, & K_{3}^{+} \mapsto K_{2}^{-}, & K_{4}^{+} \mapsto K_{1}^{-}, \\
H_{9}^{+} \mapsto H_{9}^{-} . & & &
\end{array}
$$

To see that $A$ induces a diffeomorphism between $M\left(\widetilde{F S}^{+}\right)$and $M\left(\widetilde{F S}^{-}\right)$, it suffices to show that the automorphism $A$ sends $H_{10}^{+}$to $H_{10}^{-}$.

Recall that $\gamma_{ \pm}=\frac{1}{2}(1 \pm \sqrt{5})$. One can check that for any point

$$
P:=\left[x,\left(1 / \gamma_{+}-1\right) x+z, z\right]
$$

on $H_{10}^{+}$, the image $P \cdot A$ is a point on $H_{10}^{-}$. In fact,

$$
\left(\begin{array}{lll}
x \quad\left(1 / \gamma_{+}-1\right) x+z & z
\end{array}\right) \cdot A \cdot\left(\begin{array}{c}
1 / \gamma_{-}-1 \\
-1 \\
1
\end{array}\right) \equiv 0 .
$$

Therefore, the pair $\left(\widetilde{F S}^{+}, \widetilde{F S}^{-}\right)$is not a Zariski pair.

From this example, we see that moduli spaces of fiber-type projective line arrangements do not have to be connected. In fact, we can produce infinitely many fiber-type projective line arrangements whose moduli spaces are disconnected. On the other hand, we do not know if fundamental groups of complements of fiber-type projective line arrangements are determined by intersection lattices.

\section{Acknowledgements}

The author is grateful to M. Amram, M. Cohen, M. Eliyahu, D. Garber, M. Teicher, E. Artal Bartolo, and J.I. Cogolludo Agustín for their helpful conversations and comments, and especially to D. Garber for comments on a draft of this manuscript.

\section{References}

[Amram et al. 2012] M. Amram, M. Teicher, and F. Ye, "Moduli spaces of arrangements of 10 projective lines with quadruple points", preprint, 2012. To appear in Adv. Appl. Math. arXiv 1206.2486 [Artal 1994] E. Artal Bartolo, "Sur les couples de Zariski”, J. Algebraic Geom. 3:2 (1994), 223-247. MR 94m:14033 Zbl 0823.14013

[Artal et al. 2005] E. Artal Bartolo, J. Carmona Ruber, J. I. Cogolludo-Agustín, and M. Marco Buzunáriz, "Topology and combinatorics of real line arrangements", Compos. Math. 141:6 (2005), 1578-1588. MR 2006k:32055 Zbl 1085.32012

[Artal et al. 2008] E. Artal Bartolo, J. I. Cogolludo, and H.-o. Tokunaga, "A survey on Zariski pairs", pp. 1-100 in Algebraic geometry in East Asia (Hanoi, 2005), edited by K. Konno and V. Nguyen-Khac, Adv. Stud. Pure Math. 50, Math. Soc. Japan, Tokyo, 2008. MR 2009g:14030 Zbl 1141.14015 
[Cohen and Suciu 1997] D. C. Cohen and A. I. Suciu, "The braid monodromy of plane algebraic curves and hyperplane arrangements", Comment. Math. Helv. 72:2 (1997), 285-315. MR 98f:52012 Zbl 0959.52018

[Csima and Sawyer 1993] J. Csima and E. T. Sawyer, "There exist 6n/13 ordinary points", Discrete Comput. Geom. 9:2 (1993), 187-202. MR 94a:52015 Zbl 0771.52003

[Fan 1997] K.-M. Fan, "Direct product of free groups as the fundamental group of the complement of a union of lines", Michigan Math. J. 44:2 (1997), 283-291. MR 98j:14039 Zbl 0911.14007

[Garber et al. 2003] D. Garber, M. Teicher, and U. Vishne, " $\pi_{1}$-classification of real arrangements with up to eight lines”, Topology 42:1 (2003), 265-289. MR 2004b:32048 Zbl 1074.14050

[Grünbaum 2009] B. Grünbaum, Configurations of points and lines, Graduate Studies in Mathematics 103, American Mathematical Society, Providence, RI, 2009. MR 2011j:52001 Zbl 1205.51003

[Hirzebruch 1986] F. Hirzebruch, "Singularities of algebraic surfaces and characteristic numbers", pp. 141-155 in The Lefschetz centennial conference, I (Mexico City, 1984), edited by D. Sundararaman, Contemp. Math. 58, Amer. Math. Soc., Providence, RI, 1986. MR 87j:14057 Zbl 0601.14030

[Jiang and Yau 1994] T. Jiang and S. S.-T. Yau, "Diffeomorphic types of the complements of arrangements of hyperplanes", Compositio Math. 92:2 (1994), 133-155. MR 95e:32042 Zbl 0828.57018

[Jiang and Yau 1998] T. Jiang and S. S.-T. Yau, "Intersection lattices and topological structures of complements of arrangements in CP2", Ann. Scuola Norm. Sup. Pisa Cl. Sci. (4) 26:2 (1998), 357-381. MR 99g:52020 Zbl 0973.32015

[Jiang et al. 2001] T. Jiang, S. S.-T. Yau, and L.-Y. Yeh, "Simple geometric characterization of supersolvable arrangements", Rocky Mountain J. Math. 31:1 (2001), 303-312. MR 2001m:55047 Zbl 1008.32015

[Nazir and Yoshinaga 2012] S. Nazir and M. Yoshinaga, "On the connectivity of the realization spaces of line arrangements”, Ann. Sc. Norm. Super. Pisa, Cl. Sci. 11:4 (2012), 921-937. Zbl 06142478

[Rybnikov 2011] G. L. Rybnikov, "On the fundamental group of the complement of a complex hyperplane arrangement”, Funktsional. Anal. i Prilozhen. 45:2 (2011), 71-85. MR 2012i:14067

[Wang and Yau 2005] S. Wang and S. S.-T. Yau, "Rigidity of differentiable structure for new class of line arrangements", Comm. Anal. Geom. 13:5 (2005), 1057-1075. MR 2007d:32021 Zbl 1115.52010

Received July 3, 2012. Revised November 13, 2012.

FEI YE

DEPARTMENT OF MATHEMATICS

THE University OF Hong Kong

POKFULAM

HONG KONG

fye@maths.hku.hk

http://hkumath.hku.hk/ fye 


\title{
PACIFIC JOURNAL OF MATHEMATICS
}

\author{
msp.org/pjm
}

Founded in 1951 by E. F. Beckenbach (1906-1982) and F. Wolf (1904-1989)

\section{EDITORS}

V. S. Varadarajan (Managing Editor)

Department of Mathematics

University of California

Los Angeles, CA 90095-1555

pacific@math.ucla.edu

Paul Balmer

Department of Mathematics

University of California

Los Angeles, CA 90095-1555

balmer@math.ucla.edu

Daryl Cooper

Department of Mathematics

University of California

Santa Barbara, CA 93106-3080 cooper@math.ucsb.edu

Jiang-Hua $\mathrm{Lu}$

Department of Mathematics

Pokfulam Rd., Hong Kong jhlu@maths.hku.hk
The University of Hong Kong

Don Blasius

Department of Mathematics University of California

Los Angeles, CA 90095-1555

blasius@math.ucla.edu

Robert Finn

Department of Mathematics Stanford University

Stanford, CA 94305-2125

finn@math.stanford.edu

Sorin Popa

Department of Mathematics

University of California

Los Angeles, CA 90095-1555 popa@math.ucla.edu

Paul Yang

Department of Mathematics Princeton University

Princeton NJ 08544-1000

yang@math.princeton.edu

\section{PRODUCTION}

Silvio Levy, Scientific Editor, production@msp.org

\section{SUPPORTING INSTITUTIONS}

ACADEMIA SINICA, TAIPEI

CALIFORNIA INST. OF TECHNOLOGY

INST. DE MATEMÁTICA PURA E APLICADA

KEIO UNIVERSITY

MATH. SCIENCES RESEARCH INSTITUTE

NEW MEXICO STATE UNIV.

OREGON STATE UNIV.

\author{
STANFORD UNIVERSITY \\ UNIV. OF BRITISH COLUMBIA \\ UNIV. OF CALIFORNIA, BERKELEY \\ UNIV. OF CALIFORNIA, DAVIS \\ UNIV. OF CALIFORNIA, LOS ANGELES \\ UNIV. OF CALIFORNIA, RIVERSIDE \\ UNIV. OF CALIFORNIA, SAN DIEGO \\ UNIV. OF CALIF., SANTA BARBARA
}

\author{
Vyjayanthi Chari \\ Department of Mathematics \\ University of California \\ Riverside, CA 92521-0135 \\ chari@math.ucr.edu \\ Kefeng Liu \\ Department of Mathematics \\ University of California \\ Los Angeles, CA 90095-1555 \\ liu@math.ucla.edu \\ Jie Qing \\ Department of Mathematics \\ University of California \\ Santa Cruz, CA 95064 \\ qing@cats.ucsc.edu
}

These supporting institutions contribute to the cost of publication of this Journal, but they are not owners or publishers and have no responsibility for its contents or policies.

See inside back cover or msp.org/pjm for submission instructions.

The subscription price for 2013 is US \$400/year for the electronic version, and \$485/year for print and electronic.

Subscriptions, requests for back issues and changes of subscribers address should be sent to Pacific Journal of Mathematics, P.O. Box 4163, Berkeley, CA 94704-0163, U.S.A. The Pacific Journal of Mathematics is indexed by Mathematical Reviews, Zentralblatt MATH, PASCAL CNRS Index, Referativnyi Zhurnal, Current Mathematical Publications and the Science Citation Index.

The Pacific Journal of Mathematics (ISSN 0030-8730) at the University of California, c/o Department of Mathematics, 798 Evans Hall \#3840, Berkeley, CA 94720-3840, is published twelve times a year. Periodical rate postage paid at Berkeley, CA 94704, and additional mailing offices. POSTMASTER: send address changes to Pacific Journal of Mathematics, P.O. Box 4163, Berkeley, CA 94704-0163.

PJM peer review and production are managed by EditFLOW ${ }^{\circledR}$ from Mathematical Sciences Publishers.

\section{PUBLISHED BY}

mathematical sciences publishers

nonprofit scientific publishing

http://msp.org/

(C) 2013 Mathematical Sciences Publishers 


\section{PACIFIC JOURNAL OF MATHEMATICS}

Volume $265 \quad$ No. $1 \quad$ September 2013

Genus-two Goeritz groups of lens spaces

SANGBUM CHO

A compact embedding theorem for generalized Sobolev spaces

SENG-KeE ChUa, SCOTT Rodney and Richard L. WheEden

Partial integrability of almost complex structures and the existence of

solutions for quasilinear Cauchy-Riemann equations

CHONG-KYU HAN and JONG-DO PARK

An overdetermined problem in potential theory

DMITRY KHAVINSON, ERIK LUNDBERG and RAZVAN TEODORESCU

Quasisymmetric homeomorphisms on reducible Carnot groups

XIANGDONG XIE

Capillarity and Archimedes' principle

JOHN MCCUAN and RAY TREINEN

Generalized eigenvalue problems of nonhomogeneous elliptic operators and their application

DUmitru Motreanu and Mieko TANaKa

Weighted Ricci curvature estimates for Hilbert and Funk geometries

SHIN-ICHI OHTA

On generalized weighted Hilbert matrices

EMMANUEL PREISSMANN and OLIVIER LÉVÊQUE

Unique prime decomposition results for factors coming from wreath product 221 groups

J. OWEN SizEMORE and ADAM WinCHESTER

On volume growth of gradient steady Ricci solitons

Guofang Wei and Peng Wu

Classification of moduli spaces of arrangements of nine projective lines

FEI YE 